\title{
Expression of fourteen novel obesity-related genes in zucker diabetic fatty rats
}

\author{
Peter M Schmid ${ }^{1,4^{*}}$, Iris Heid ${ }^{2}$, Christa Buechler ${ }^{3}$, Andreas Steege ${ }^{1}$, Markus Resch', Christoph Birner ${ }^{1}$,
}

Dierk H Endemann ${ }^{1}$, Guenter A Riegger ${ }^{1}$ and Andreas Luchner ${ }^{1}$

\begin{abstract}
Background: Genome-wide association studies (GWAS) are useful to reveal an association between single nucleotide polymorphisms and different measures of obesity. A multitude of new loci has recently been reported, but the exact function of most of the according genes is not known. The aim of our study was to start elucidating the function of some of these genes.

Methods: We performed an expression analysis of fourteen genes, namely BDNF, ETV5, FAIM2, FTO, GNPDA2, KCTD15, LYPLAL1, MCR4, MTCH2, NEGR1, NRXN3, TMEM18, SEC16B and TFAP2B, via real-time RT-PCR in adipose tissue of the kidney capsule, the mesenterium and subcutaneum as well as the hypothalamus of obese Zucker diabetic fatty (ZDF) and Zucker lean (ZL) rats at an age of 22 weeks.

Results: All of our target genes except for SEC16B showed the highest expression in the hypothalamus. This suggests a critical role of these obesity-related genes in the central regulation of energy balance. Interestingly, the expression pattern in the hypothalamus showed no differences between obese ZDF and lean ZL rats. However, LYPLAL1, TFAP2B, SEC16B and FAIM2 were significantly lower expressed in the kidney fat of ZDF than ZL rats. NEGR1 was even lower expressed in subcutaneous and mesenterial fat, while MTCH2 was higher expressed in the subcutaneous and mesenterial fat of ZDF rats.

Conclusion: The expression pattern of the investigated obesity genes implies for most of them a role in the central regulation of energy balance, but for some also a role in the adipose tissue itself. For the development of the ZDF phenotype peripheral rather than central mechanisms of the investigated genes seem to be relevant.
\end{abstract}

Keywords: BDNF, ETV5, FAIM2, FTO, GNPDA2, KCTD15, LYPLAL1, MCR4, MTCH2, NEGR1, NRXN3, TMEM18, SEC16B, TFAP2B, ZDF-rats

\section{Background}

By now, overweight and obesity have become a major public health problem in the industrialized world and also in developing countries. Both result in an increased morbidity and mortality, not to mention the financial costs for the health-care systems. According to the World Health Organisation (WHO) in 2005 worldwide at least 400 million adults were obese and this count is expected to rise to 700 million until 2015 (http://www. who.int/gho/ncd/risk_factors/overweight/en/). Thereby,

\footnotetext{
* Correspondence: peterm.schmid@ukr.de

${ }^{1}$ Klinik und Poliklinik für Innere Medizin II, University of Regensburg,

Regensburg, Germany

${ }^{4}$ Klinik und Poliklinik für Innere Medizin II, Franz-Josef-Strauss-Allee 11,

University of Regensburg, 93042 Regensburg, Germany

Full list of author information is available at the end of the article
}

lifestyle changes with an increased intake of energydense foods and a trend to decreased physical activity are fundamental causes for this rise to epidemic proportions, but heritability studies provide evidence for a notable genetic involvement $[1,2]$.

Genome-wide association studies (GWAS) are excellently qualified to reveal single genetic polymorphisms associated with human obesity, but they allow no conclusions about the function of the affected genes. Since recent GWAS [3-11] in adults could find different genetic loci located next to or in so far partly unknown genes, now the physiologic role of most of these genes has to be elucidated.

The aim of our study was to shed some light in the role of fourteen recently described genes, namely BDNF, 
ETV5, FAIM2, FTO, GNPDA2, KCTD15, LYPLAL1, MCR4, MTCH2, NEGR1, NRXN3, TMEM18, SEC16B, and TFAP2B, which were associated with different measures of human obesity. Thereby, as a first step it is reasonable to identify tissues, in which those genes are expressed, and to reveal differences in the expression levels between obese and lean subjects. Therefore our study was planned as a descriptive expression analysis of these obesity-related genes in an animal model of diabetes mellitus type 2 and obesity, the ZDF rat, in comparison to their lean normoglycemic littermate, the ZL rat.

Some of the obesity-genes are already known to regulate energy homeostasis and for some others it is shown that they are expressed in the central nervous system [11] and may influence energy expenditure centrally in the hypothalamus. For other genes functions apart from the regulation of energy homeostasis are described and for some genes their function is totally unknown. Since the adipose tissue is not only a simple energy storage, but also a key regulator of body weight $[12,13]$, it is also a possible location of action for these obesity-related genes. Thereby, it is important to differentiate between adipose tissues of different origin as it is known that especially mesenterial adipose tissue harbors a high risk for comorbidities like diabetes mellitus type 2 and atherosclerosis [14]. Therefore the gene expression was measured via real-time RT-PCR in the hypothalamus as regulatory center of energy balance and food intake [15] and in the adipose tissue of the mesenterium, the subcutaneum and the kidney capsule.

\section{Methods}

\section{Animal experiment}

The study was approved by the local committee on animal research and is in accordance with the "Guide for the Care and Use of Laboratory Animals' published by the US National Institutes of Health. The EU legislation for animal care (86/609/CEE) was applied. Six male Zucker diabetic fatty (ZDF) rats (fa/fa) and six male Zucker lean rats (fa/-) (ZL) were studied from an age of 13 weeks until an age of 24 weeks. The animals were individually housed on a 12-hour dark/12-hour light cycle. They were fed a Purina 5008 rat chow containing 23\% protein, $6.5 \%$ fat, $58.5 \%$ carbohydrates, $4 \%$ fiber and $8 \%$ ash. Rats received tap water ad libitum. In week 14 and 22 systolic blood pressure was assessed by tail cuff method using an automated cuff inflator-pulse detection system (CODA2 Multi-Channel, Computerized, EMKA TECHNOLOGIES, Paris, France). At the same timepoints the animals were weighted and blood glucose was measured after 6 hours fasting (ACCU-CHEK Sensor, Roche $\mathrm{GmbH}$, Mannheim, Germany). Animals were sacrificed at 24 weeks of age and we prepared the adipose tissue of the mesenterium (mesenterial fat, MF), the kidney capsule (kidney fat, KF) and subcutaneum (subcutaneous fat, SF) as well as the hypothalamus (HT) of the six ZDF and ZL rats. Serum levels of leptin and adiponectin as marker of obesity were determined via ELISA according to the manufacturers instructions (B-Bridge International, Inc., 5 Mountain View, USA).

\section{Expression analysis via real-time RT-PCR}

The fourteen target genes were chosen according to the results of recent GWAS and metaanalysis of GWAS [4-11], in which they were found to be significantly associated with different measures of obesity. The gene expression was measured in three different adipose tissues (KF (kidney fat), SF (subcutaneous fat) and MF (mesenterial fat)) and the hypothalamus (HT) by using the realtime RT-PCR technique. Therefore total RNA from the different tissues was extracted using the RNeasy kit (Quiagen, Hilden, Germany) according to the manufacturers instructions. A DNase digestion step was included. For first-strand cDNA synthesis, $1 \mu \mathrm{g}$ total RNA was reverse transcribed with $1 \mathrm{U}$ MMLV Reverse Transcriptase, $1 \mu \mathrm{g}$ Random Primer, $1 \mathrm{mM}$ deoxynucleotide triphosphate mixture, $1 \mu \mathrm{l}$ recombinant RNasin ${ }^{\circledR}$ ribonuclease inhibitor and transcription buffer with $5 \mathrm{mM} \mathrm{MgCl} 2$ in a final volume of $10 \mu \mathrm{l}$ (all from Promega, Mannheim, Germany). The reaction mixture was incubated at $37^{\circ} \mathrm{C}$ for $60 \mathrm{~min}$, followed by heat inactivation of the enzyme at $95^{\circ} \mathrm{C}$ for $5 \mathrm{~min}$. After cooling on ice for $5 \mathrm{~min}$, the cDNA was stored at $-20^{\circ} \mathrm{C}$ until further use. Real-time RT-PCR detection of the target genes and beta-actin as housekeeping gene was perfomed using the AbiPrism 7900 TaqMan (Applied Biosystems, Foster City; CA, USA). Primers and probes (Table 1) were used according to the manufacturer's protocol (Applied Biosystems). The expression levels of the target mRNA were normalized to beta-actin using the DeltaCt method. Parallelism of amplification curves of the target and control was confirmed.

\section{Statistical analysis}

The means of every measurement for the biometric data of $\mathrm{ZL}$ and $\mathrm{ZDF}$ rats as well as the means of the expression data from the real-time RT-PCR were calculated for each tissue sample and animal group. Groups were compared using the Students $t$ test or one-way ANOVA as appropriate. A $p$-value of $<0.05$ was considered to be significant. The biometric data are presented as means \pm SEM, the expression data are depicted in graphics with an arbitrary logarithmic y-axis as means + StdDev.

\section{Results and discussion}

\section{Biometric data of ZL and ZDF rats}

In Table 2 the biometric data of ZL and ZDF rats at an age of 22 weeks are summarized. As expected, ZDF rats 
Table 1 Primer and probes

\begin{tabular}{lll}
\hline Gene symbol & \multicolumn{1}{c}{ Gene name } & $\begin{array}{c}\text { Applied biosystems } \\
\text { assay ID }\end{array}$ \\
\hline Actb & Beta Actin & Rn00667869_m1 \\
\hline BDNF & Brain derived neurotrophic factor & Rn02531967_s1 \\
\hline ETV5 & Ets variant 5 & Rn00465814_g1 \\
\hline FAIM2 & Fas apoptotic inhibitory molecule 2 & Rn01532650_m1 \\
\hline FTO & FTO & Rn01538183_m1 \\
\hline GNPDA2 & Glucosamine-6-phosphate & Rn01413702_m1 \\
\hline LYPLAL1 & Lysophospholipase-like 1 & Rn01411856_g1 \\
\hline MC4R & Melanocortin 4 receptor & Rn01491866_s1 \\
\hline MTCH2 & Mitochondrial carrier homolog 2 & Rn01013168_m1 \\
\hline NRXN3 & Neurexin 3 & Rn00587546_m1 \\
\hline NEGR1 & Neuronal growth regulator 1 & Rn00572380_m1 \\
\hline KCTD15 & Potassium channel tetramerisation & Rn01458149_m1 \\
\hline SEC16B & SEC16 homolog B & Rn00585728_m1 \\
\hline TFAP2B & Transcription factor AP-2 beta & Rn01511928_m1 \\
\hline TMEM18 & Transmembrane protein 18 & Rn01473465_g1 \\
\hline
\end{tabular}

demonstrated diabetes mellitus type 2 with a significantly elevated blood glucose, while the ZL rats were normoglycemic. The blood pressure was lower in the ZDF rats in terms of an advanced stage of disease. Surprisingly, the ZL rats weighted significantly more than the diabetic ZDF rats, but that was due to a better growth during the animal experiment, which was represented in a significantly greater tibia length in ZL rats. However the ZDF rats apparently had a different body composition with a smaller size and a higher body fat portion. This is also reflected by significantly higher serum levels of leptin and lower serum levels of adiponectin in ZDF rats.

\section{Genes with a known role in regulation of energy homeostasis}

FTO (fat mass and obesity associated, Figure 1a) was the first gene of the GWAS era, which was found to be associated with obesity [16], and therefore is one of the best investigated obesity-gene [17]. It is highly expressed in the arcuate nucleus of the hypothalamus and is supposed to have an anorexigenic function since an overexpression of FTO in the hypothalamus decreases and a knockdown of FTO increases food intake [18]. In our study the greatest amount of FTO mRNA in both animals was detected in the hypothalamus with significant differences to the other tissues (ZL and ZDF: $p<0.001$ for HT vs KF, SF and MF). Only in ZL rats the expression in subcutaneous fat was significantly lower than in kidney and mesenterial fat $(p=0.001$ for SF vs KF, $p=0.037$ for SF vs $\mathrm{MF}$ ), in ZDF rats the expression in the fat tissues was equally high. Significant changes between the animal groups for a single tissue could not be detected.
MC4R (melanocortin 4 receptor, Figure $1 \mathrm{~b}$ ) as part of the central melanocortin system is highly expressed in the hypothalamus and mutations in the gene are thought to be a reason for obesity [19]. $\alpha$-melanocyte stimulating hormone binds to MC4R in the hypothalamus, which leads to an enhancement of anorexigenic and an inhibition of orexigenic stimuli [20]. MC4R knock-out mice show the phenotype of the so called melanocortin obesity syndrome consisting of hyperphagia, hypometabolism, hyperinsulinemia and increased linear growth $[21,22]$. Generally, the central melanocortin system is accepted to be a key regulator of energy homeostasis and food intake, it affects body composition and diverse signals for the regulation of energy homeostasis like leptin interact with it [23]. The expression of MC4R showed the highest levels in the hypothalamus and mesenterial fat of ZL as well as ZDF rats. In kidney and subcutaneous fat the amount was significantly lower than in the hypothalamus and mesenterial fat (ZL: $p<0.001$ for MF vs. KF, $p=0.001$ for MF vs. SF, $p<0.001$ for HT vs. KF, $p=0.002$ for HT vs. SF; ZDF: $p=0.007$ for MF vs. KF, $p=0.009$ for MF vs. SF, $p<0.001$ for HT vs. KF and SF). Comparing each single tissue between $\mathrm{ZL}$ and ZDF rats no significant difference could be found.

BDNF (brain derived neurotrophic factor, Figure 1c) as nerve growth factor is widely distributed throughout the whole brain [24]. Recently, BDNF was also suggested to be an important effector of the melanocortin system since BDNF infusion in the brain suppressed hyperphagia and weight gain in MC4R deficient mice [25]. For BDNF the expression level was similar high in the hypothalamus and mesenterial fat of ZL and ZDF rats each with significant differences to the other fat tissues (ZL and ZDF: $p<0.001$ for HT vs. KF and SF, $p<0.001$ for HT vs. MF). A significant difference between kidney fat and subcutaneous fat was not detected in both animal groups. Also, there were no significant differences between ZL and ZDF rats for a special tissue.

In summary, our data support the known role of these genes in the central regulation of energy balance even in

Table 2 Biometric data of ZL and ZDF rats at an age of 22 weeks

\begin{tabular}{|c|c|c|c|}
\hline \multirow[t]{2}{*}{ Parameters } & \multicolumn{3}{|c|}{ Week 22} \\
\hline & $\overline{Z L}(n=6)$ & ZDF $(n=6)$ & $p$ \\
\hline Blood glucose $[\mathrm{mg} / \mathrm{dl}]$ & $87 \pm 1$ & $393 \pm 19$ & $<0.001$ \\
\hline $\mathrm{SBP}[\mathrm{mmHg}]$ & $119 \pm 5$ & $99 \pm 2$ & 0.003 \\
\hline Body weight [g] & $405 \pm 9$ & $376 \pm 4$ & 0.01 \\
\hline Tibia length $[\mathrm{cm}]$ & $4.5 \pm 0.04$ & $4.3 \pm 0.03$ & 0.008 \\
\hline Leptin [ng/ml] & $10.3 \pm 0.6$ & $15.4 \pm 1.6$ & 0.013 \\
\hline Adiponectin [ $\mathrm{\mu g} / \mathrm{ml}]$ & $23.0 \pm 1.8$ & $15.7 \pm 0.6$ & 0.003 \\
\hline
\end{tabular}

Values are mean \pm SEM. SBP indicates systolic blood pressure. 


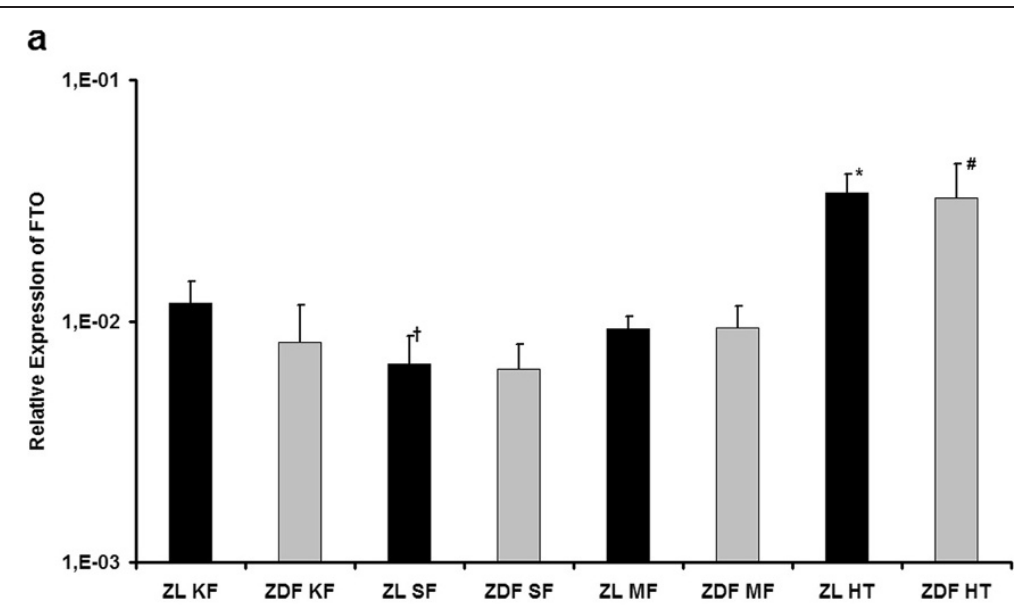

b

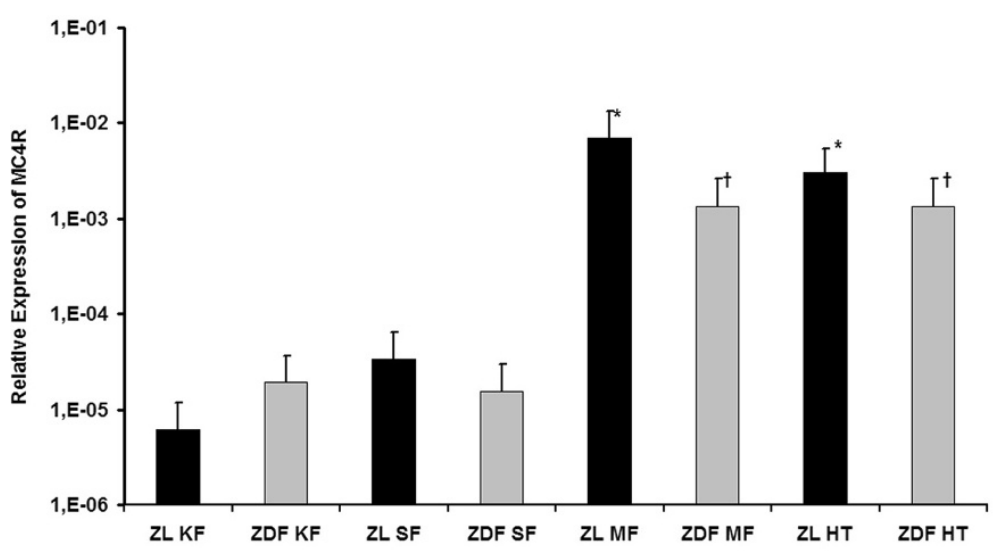

C

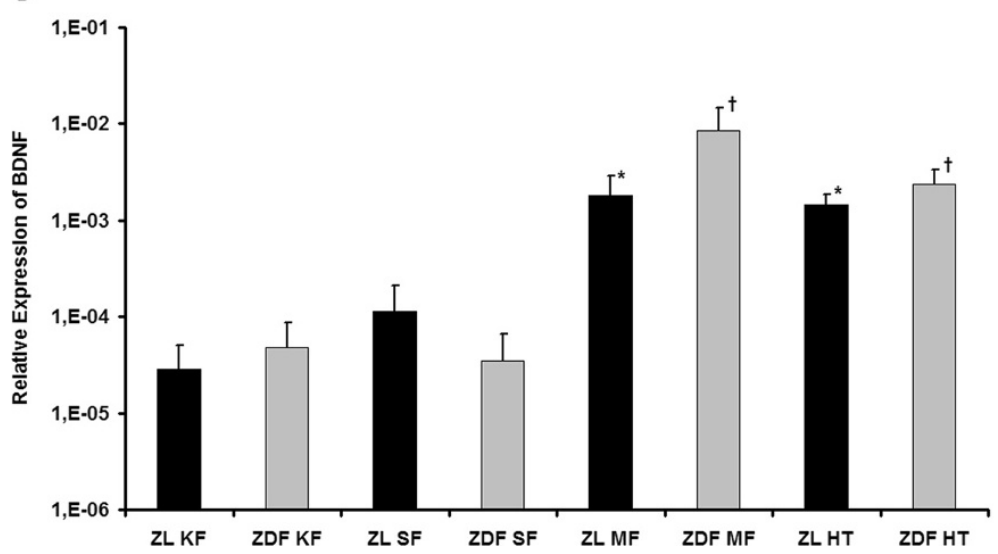

Figure 1 Expression of genes with a known role in regulation of energy homeostasis. a) Relative expression of FTO. ${ }^{*} p<0.05 \mathrm{vs}$. ZL KF, ZL SF and ZL MF; $\uparrow p<0.05$ vs. ZL KF, ZL MF and ZL HT; \# $p<0.05$ vs. ZDF KF, ZDF SF and ZDF MF. b) Relative expression of MC4R. ${ }^{*} p<0.05$ vs. ZL KF, ZL SF and ZL MF; $+p<0.05$ vs. ZDF KF, ZDF SF and ZDF MF. c) Relative expression of BDNF. ${ }^{*} p<0.05$ vs. ZL KF and ZL SF; † $p<0.05$ vs. ZDF KF and ZDF SF.

ZL and ZDF rats, but the high expression of MC4R and BDNF in the mesenterial fat suggests also a possible direct action of the melanocortin system in the adipose tissue.

\section{Genes with a known neuronal expression}

TMEM18 (transmembrane protein 18, Figure 2a) was recently found to be remarkably conserved across different species and to be expressed in many brain sites without 


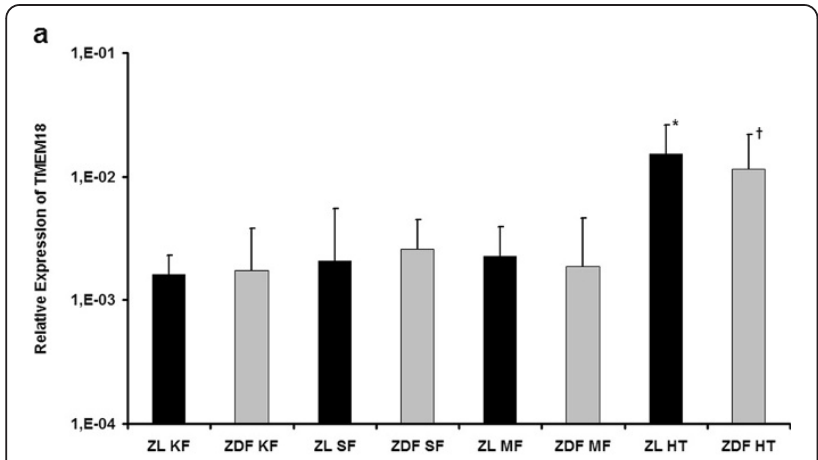

b

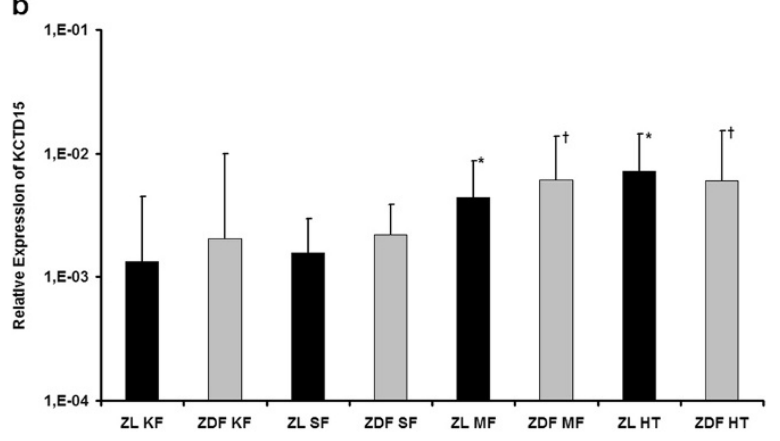

c

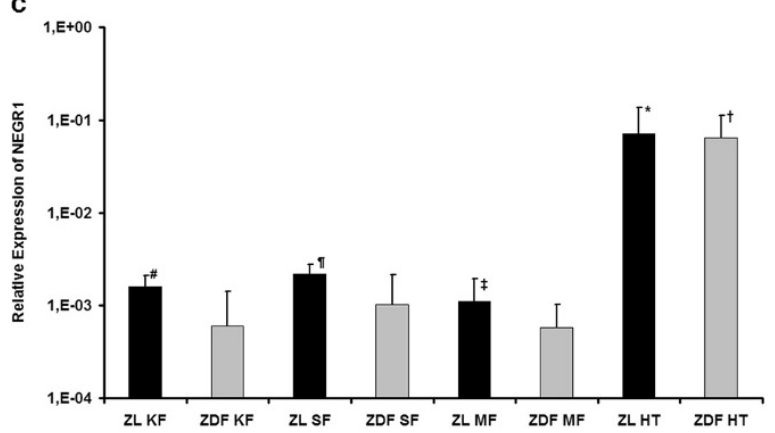

d

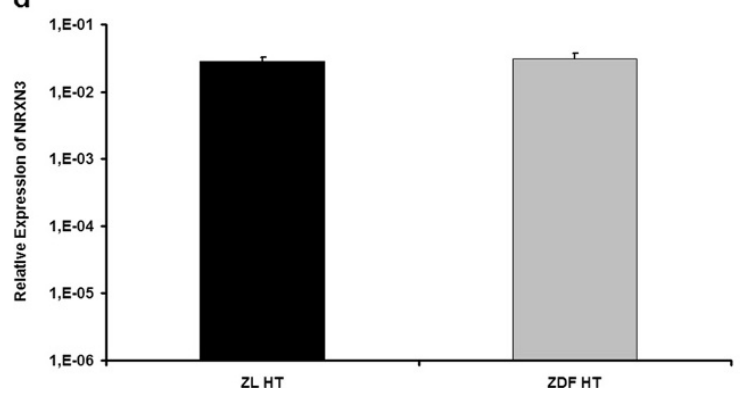

Figure 2 Expression of genes with a known neuronal expression. a) Relative expression of TMEM18. ${ }^{*} p<0.05$ vs. ZL KF, ZL SF and ZL MF; $† p<0.05$ vs. ZDF KF, ZDF SF and ZDF MF. b) Relative expression of KCTD15. ${ }^{*} p<0.05$ vs. ZL KF and ZL SF; † $p<0.05$ vs. ZDF KF and ZDF SF. c) Relative expression of NEGR1. ${ }^{*} p<0.05$ vs. ZL KF, ZL SF and ZL MF; † $p<0.05$ vs. ZDF KF, ZDF SF and ZDF MF; \# $p<0.05$ vs. ZDF KF; $p<0.05$ vs. ZDF SF; $\neq p<0.05$ vs. ZDF MF. d) Relative expression of NRXN3. No detectable mRNAamount in adipose tissues. changes between feeding-related mouse models [26]. The expression pattern of TMEM18 in ZL and ZDF rats was similar without significant differences between each single tissues of the two animal groups. The highest amount of TMEM18 mRNA was found in the hypothalamus of both animals with significant differences to the different fat tissues (ZL and ZDF: $p<0.001$ for HT vs KF, SF and MF).

KCTD15 (potassium channel tetramerisation domain containing 15, Figure $2 \mathrm{~b}$ ) is a BTB domain-containing protein and inhibits neural crest induction [27]. The highest quantitiy of KCTD15 mRNA was found for ZL and ZDF rats in mesenterial fat and the hypothalamus. Herein, the quantity was significantly higher than in kidney and subcutaneous fat (ZL: $p<0.001$ for MF and HT vs KF and SF; ZDF: $p=0.008$ for MF vs KF, $p=0.012$ for MF vs SF, $p=0.009$ for HT vs KF, $p=0.013$ for HT vs SF). Significant differences for each single tissues between the two animal groups were not detected.

NEGR1 (neuronal growth regulator 1, neurotractin, Figure 2c) seems to play an important role in neuronal outgrowth during neurogenesis $[28,29]$. For both animal groups the quantity of NEGR1 mRNA was highest in the hypothalamus (ZL and ZDF: $p<0.001$ for HT vs. KF, SF and MF). Interestingly, there were also significant differences between all three adipose tissues of ZL and ZDF rats. In kidney, subcutaneous and mesenterial fat the expression was significantly higher in ZL than in ZDF rats (KF: $p=0.002$ for ZL vs. ZDF, SF: $p=0.027$ for ZL vs ZDF, MF: $p=0.013$ for ZL vs. ZDF).

NRXN3 (neurexin 3, Figure 2d) seems to play an important role in psychiatric disorders and recently it was also found to be expressed in other tissues like the heart [30]. The only tissue, in which mRNA of NRXN3 was detectable, was the hypothalamus of ZL and ZDF rats with no significant differences between the two animal groups.

Summing up, for TMEM18, KCTD15, NEGR1 and NRXN3 a neuronal expression is known and therefore a potential central effect on energy homeostasis is possible, but so far not yet described. Our data are in line with previous findings of the central expression of these genes. Recently, for NEGR1 additionally a differential expression between obese and lean siblings in subcutaneous fat was described and a central function in an obesity-related transcript network was suggested [31]. In our work, we could also show significant changes between obese and lean rats not only in the subcutaneous fat, but also in the kidney and mesenterial fat. This implicates a possible role of NEGR1 in the regulation of obesity in the adipose tissue itself and especially in the development of the ZDF phenotype.

\section{Genes with functions so far apart from the regulation of energy homeostasis}

Mutations of TFAP2B (transcription factor activating enhancer binding protein 2 beta, Figure $3 a$ ) are known 
a

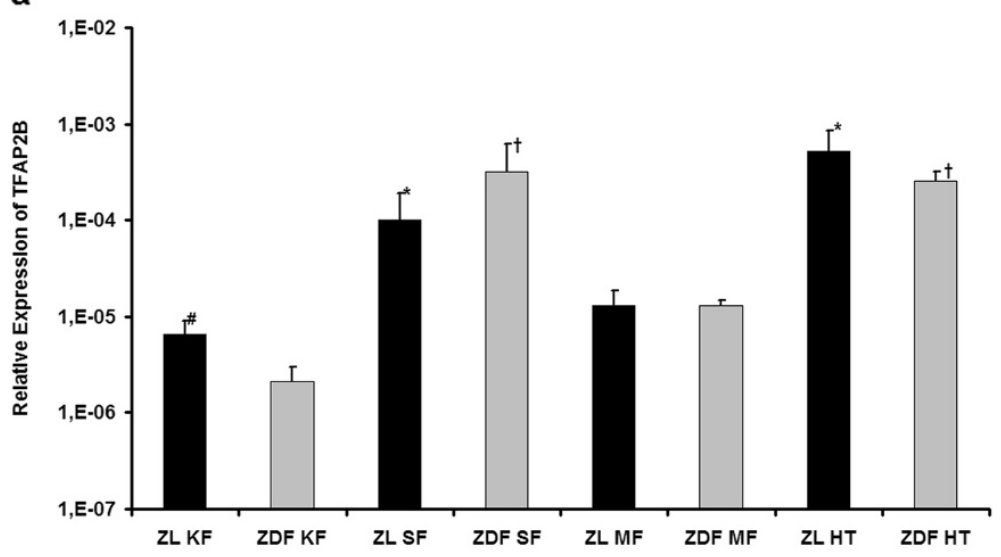

b

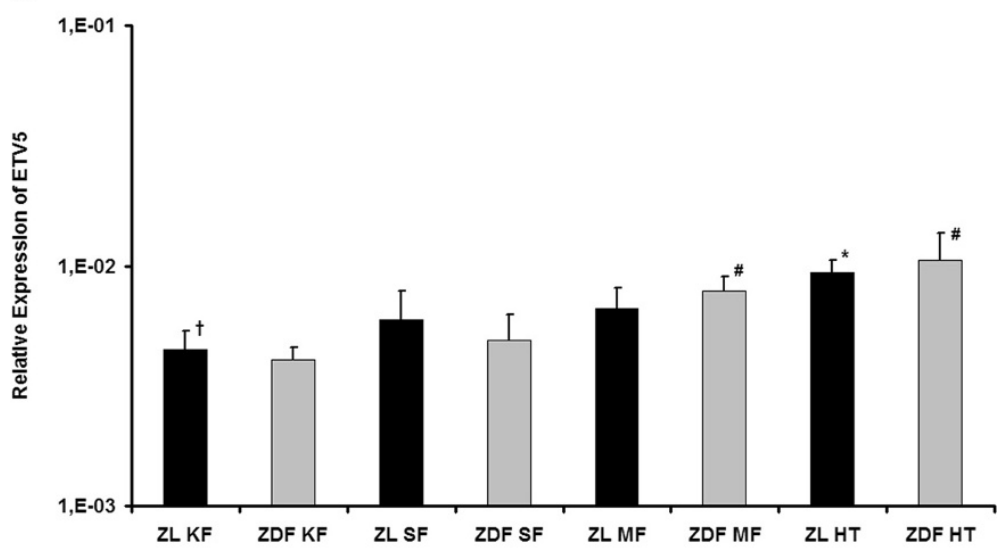

C

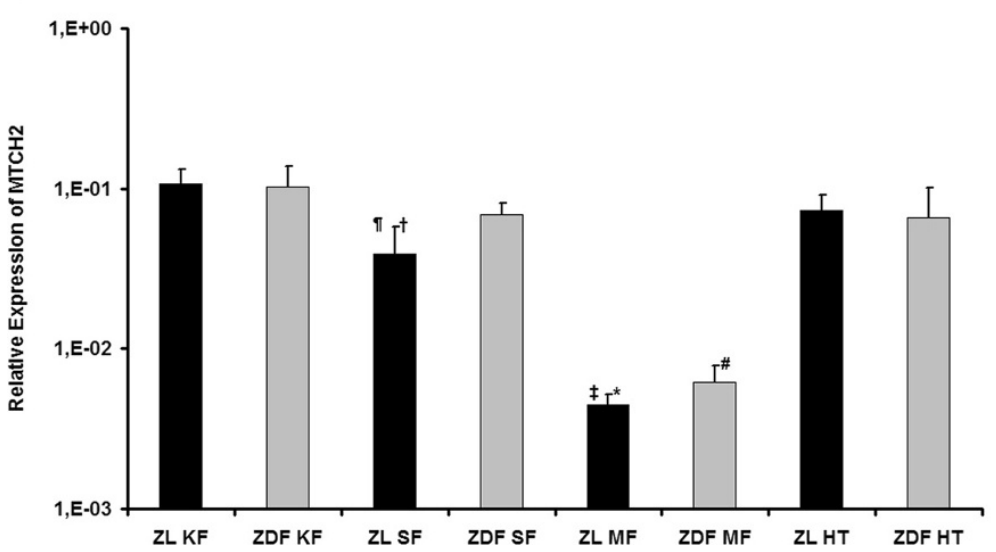

Figure 3 Expression of genes with functions so far apart from the regulation of energy homeostasis. a) Relative expression of TFAP2B. ${ }^{*} p<0.05$ vs. ZL KF and ZL MF; $\uparrow p<0.05$ vs. ZDF KF and ZDF MF; \# $p<0.05$ vs. ZDF KF. b) Relative expression of ETV5. ${ }^{*} p<0.05$ vs. ZL KF, ZL SF and ZL MF; $† p<0.05$ vs. ZL HT and ZL MF; \# $p<0.05$ vs. ZDF KF and ZDF SF. c) Relative expression of MTCH2. ${ }^{*} p<0.05$ vs. ZL KF, ZL MF and ZL $H T ; \dagger p<0.05$ vs. ZL KF and ZL HT; \# $p<0.05$ vs. ZDF KF, ZDF SF and ZDF HT; $\uparrow<0.05$ vs. ZDF SF; $\$ p<0.05$ vs. ZDF MF.

to cause the Char syndrome [32,33]. But recent studies could also show an expression in visceral and subcutaneous fat without a correlation to the body mass index or risk of diabetes mellitus type 2 in humans [34]. However, a negative correlation with the expression of adiponectin and leptin was found, which suggests that TFAP2B may 
regulate the expression of adipokines [34,35]. In our study the expression pattern of TFAP2 was similar between ZL and ZDF rats, each with highest expression in the hypothalamus and subcutaneous fat (ZL: $p<0.001$ for HT vs. KF and MF, $p=0.003$ for SF vs. KF, $p=0.021$ for SF vs. MF; ZDF: $p<0.001$ for HT vs. KF and MF, $p$ $<0.001$ for SF vs. KF and MF). In ZL rats the difference between hypothalamus and subcutaneous fat $(p=0.055)$ and in ZDF rats the difference between kidney and mesenterial fat $(p=0.051)$ did barely not reach the significance level. Comparing each single tissue between ZL and ZDF rats a significant higher expression level was only found in the kidney fat for lean animals. So, our results confirm the expression of TFAP2B in adipose tissues especially the subcutaneous fat, where it was equally high expressed as in the hypothalamus. Additionally, we could find that the expression of TFAP2B was not different between obese ZDF and lean ZL rats in the mesenterial and subcutaneous fat, but was significantly lower in the kidney fat of ZDF rats.

ETV5 (ets variant 5, Figure 3b) was until now mainly known to be an essential transcription factor for the establishment of the spermatogonial stem cell pool and its subsequent self-renewal and maintenance [36]. The expression pattern in our study of ZL and ZDF rats was quite similar without significant differences between the animal models for each tissue. Overall the highest expression was detected in the hypothalamus. In ZL rats the difference between hypothalamus and the adipose tissues was significantly $(p<0.001$ for HT vs. KF, $p=0.007$ for HT vs. SF, $p=0.037$ for HT vs. MF) and also the expression in mesenterial fat was higher than in kidney fat $(p=0.018)$. For ZDF rats the amount of ETV5 mRNA in the hypothalamus was significantly greater than in the kidney fat and subcutaneous fat $(p<0.001$ for HT vs. KF and SF), but slightly missed the significance level versus mesenterial fat $(p=0.058)$. In mesenterial fat it was significantly higher expressed than in kidney and subcutaneous fat $(p<0.001$ for MF vs. KF, $p=0.005$ for MF vs. SF). Herewith, our results reveal a so far not described high expression of ETV5 in the hypothalamus, which suggests a possible role in the central regulation of energy balance.

MTCH2 (mitochondrial carrier 2, Figure 3c) is a mitochondrial membrane protein and seems to regulate cell proliferation and apoptosis [37,38]. Lately, a high expression of MTCH2 was described in adipose tissue. It could be shown that the expression was higher in subcutaneous fat than in visceral fat and that the expression of MTCH2 was higher in obese than in lean women [39]. In our investigation the expression of $\mathrm{MTCH} 2$ was lowest in mesenterial fat of ZL and ZDF rats (ZL and ZDF: $p$ $<0.001$ for MF vs. KF, SF and HT). In ZDF animals the expression in the other three tissues was similar high, only in ZL rats the expression in the subcutaneous fat was lower than in the kidney fat and the hypothalamus $(p<0.001$ for SF vs. KF, $p=0.001$ for SF vs HT). In comparison of each single tissue between lean and obese animals the expression of MTCH2 in the subcutaneous $(p=0.017)$ and the mesenterial fat $(p=0.014)$ was significantly higher in ZDF rats. Therewith, our data are in line with aforementioned studies showing a higher expression of $\mathrm{MTCH} 2$ in the subcutaneous fat than in mesenterial fat und also finding a higher expression in obese ZDF rats than in lean ZL rats. However, a new finding is the high expression in the hypothalamus of both animal groups. Therefore $\mathrm{MTCH} 2$ may play an important role in central and peripheral regulation of obesity.

\section{Genes with unknown functions}

The function of the GWAS obesity-related genes GNPDA2, FAIM2 and LYPLAL1 is to our knowledge mainly unkown and we report here the first expression data for these genes in comparison between obese and lean animals.

The expression of GNPDA2 (glucosamine 6 phosphate deaminase 2, Figure 4a) was for both animal models highest in the hypothalamus with significant differences to the fat tissues (ZL: $p<0.001$ for HT vs. KF, SF and MF; ZDF: $p=0.008$ for HT vs. KF, $p=0.001$ for HT vs. SF, $p=0.006$ for HT vs MF). The expression in kidney, subcutaneous and mesenterial fat was at similar levels with no differences between the ZL and ZDF rats.

For FAIM2 (fas apoptotic inhibitory molecule 2, Figure $4 \mathrm{~b}$ ) the highest expression was observed for obese and lean animals in the hypothalamus with significant differences to the various fat tissues (ZL and ZDF: $p<0.001$ for HT vs. KF, SF and MF). Whereas for ZDF rats the expression in the fat tissues was at the same level, it was significantly lower in mesenterial fat of ZL rats in comparison to the other fat tissues $(p=0.023$ for MF vs. SF, $p=0.032$ for MF vs. KF). Comparing each single tissues between both animal groups only in kidney fat a significantly decrease was detected in ZDF rats $(p=0.021)$.

Looking at ZL rats, the lowest expression of $\mathbf{L Y P L A L 1}$ (lysophospholipase like 1, Figure 4c) was found in mesenterial fat with significant differences to all other tissue samples $(p<0.001$ for MF vs. SF, KF and HT). Highest levels were seen in the hypothalamus and kidney fat ( $p<0.001$ for HT vs. SF, $p<0.001$ for KF vs. SF). In ZDF rats the expression pattern was quite similar, only the expression in kidney fat was significantly lower than in ZL rats $(p=0.017)$, which resulted in a similar quantity to that of subcutaneous fat $(p=0.026$ for MF vs. SF, $p=0.012$ for MF vs. KF, $p<0.001$ for MF vs $\mathrm{HT}$, $p=0.020$ for HT vs SF, $p=0.045$ for HT vs. KF).

For SEC16B (SEC16 homolog B, Figure 4d) in ZL rats the highest expression was found in the subcutaneous fat 
a

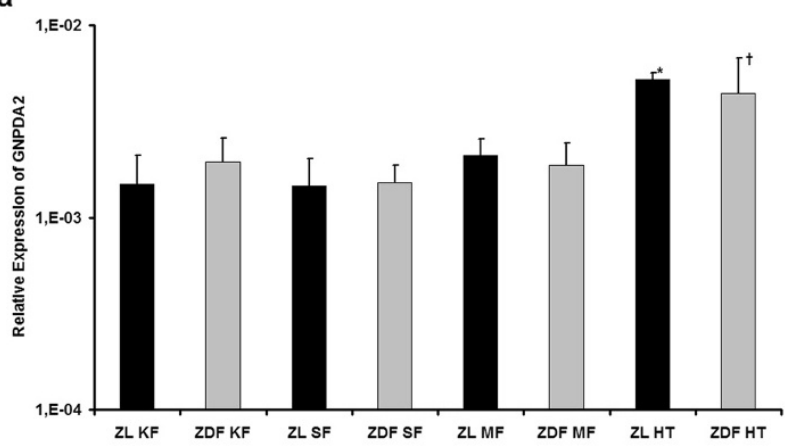

b

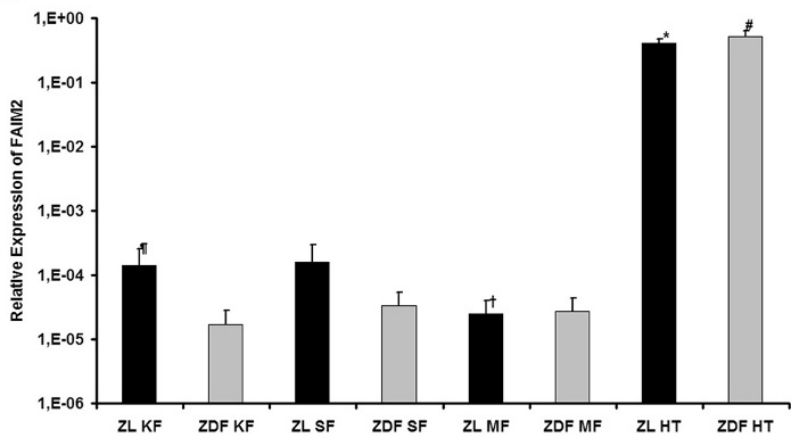

C

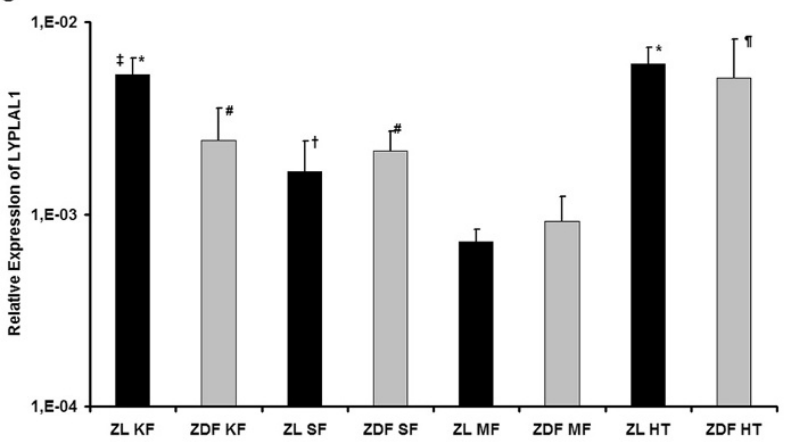

d

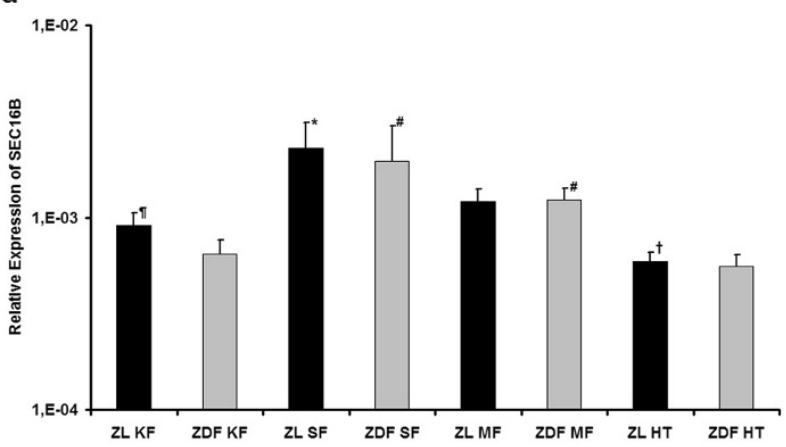

Figure 4 Expression of genes with unknown functions. a) Relative expression of GNPDA2. ${ }^{*} p<0.05$ vs. ZL KF, ZL SF and ZL MF; † $p<0.05$ vs. ZDF KF, ZDF SF and ZDF MF. b) Relative expression of FAIM2. ${ }^{*} p<0.05$ vs. ZL KF, ZL SF and ZL MF; † $p<0.05$ vs. ZL KF, ZL MF and ZL HT; \# $p<0.05$ vs. ZDF KF, ZDF SF and ZDF MF; $p<0.05$ vs. ZDF KF. c) Relative expression of LYPLAL1. ${ }^{*} p<0.05$ vs. ZL SF and ZL MF; † $p<0.05$ vs. ZL KF, ZL MF and ZL HT; \# $p<0.05$ vs. ZDF MF and ZDF HT; $\uparrow<0.05$ vs. ZDF KF, ZDF SF and ZDF MF; $\$ p<0.05$ vs ZDF KF. d) Relative expression of SEC16B. ${ }^{*} p<0.05$ vs. ZL KF, ZL MF and ZL HT; $\dagger p<0.05$ vs. ZL KF and ZL MF; \# $p<0.05$ vs. ZDF MF and ZDF HT; $p<0.05$ vs. ZDF KF. 
with significant differences to the other tissues $(p<0.001$ for SF vs. MF, KF and HT) and lowest in the hypothalamus with significant differences to the kidney and mesenterial fat ( $p=0.008$ for HT vs KF, $p<0.001$ for HT vs. MF). In ZDF rats the pattern was quite similar ( $p$ $<0.001$ for SF vs. KF and HT, $p=0.003$ for MF vs. HT, $p=0.011$ for MF vs KF), although the difference between subcutaneous and mesenterial fat slightly failed the significance level $(p=0.059)$. In the kidney fat the expression in ZL rats was significantly higher than in the ZDF rats $(p=0.010)$.

Taking together, whereas the high expression of these genes with unknown function in the hypothalamus again suggests a role in central energy regulation, LYPLAL1 with its notable expression in the kidney fat and the differences between ZDF and ZL rats implicates also a role in the adipose tissue itself. SEC16B was the only investigated gene that showed the highest expression not in the hypothalamus. Its function is mainly unknown, but it was highest expressed in the subcutaneous fat alone, which indicates a possible role in the peripheral regulation of obesity.

In summary, our results show that all of the fourteen target genes are expressed in the hypothalamus and in the investigated adipose tissues. The only exception was NRXN3, which was exclusively expressed in the hypothalamus and not in the adipose tissues. Likewise, TMEM18, NEGR1, GNPDA2, FTO, FAIM2 and ETV5 showed the highest expression in the hypothalamus. The highest expression for MC4R, KCTD15, BDNF, LYPLAL1, MTCH2 and TFAP2B was also observed in the hypothalamus, but the level was similar high for MC4R, KCTD15 and BDNF in the mesenterial fat, for LYPLAL1 and MTCH2 in the kidney fat and for TFAP2B in the subcutaneous fat. SEC16B was the only gene, which showed the highest expression not in the hypothalamus, but in the subcutaneous fat alone. Concomitant the expression of SEC16B was lowest in the hypothalamus and kidney fat. This fact is in line with recent results from the GIANT consortium, which also found a high expression for a bigger part of the obesityrelated genes in the hypothalamus [11] and suggests a critical role of these GWAS obesity associated genes in the central regulation of energy balance.

Despite this fact it is very interesting that there were no significant differences between the target genes in the hypothalamus of ZL and ZDF rats. Differences were observed in the kidney fat, where the expression of LYPLAL1, TFAP2B, NEGR1, SEC16B and FAIM2 was significantly lower in the ZDF rats. Additionally for NEGR1 a significantly lower expression was found in all three adipose tissues of ZDF rats and for MTCH2 a significantly higher expression was found in the subcutaneous and mesenterial fat of ZDF rats. This suggests that putative important mechanisms of the obesity associated genes in the hypothalamus are not responsible for the development of obesity in ZDF rats. Instead changes in the adipose tissue seem to be accountable for the development of the ZDF phenotype.

Taking further into account that ZDF rats exhibit hyperleptinemia (see Table 2) due to a defect in the leptin receptor, we can assume that the actions of those genes without differential expressions between ZL and $\mathrm{ZDF}$ rats must be rather independent of leptin levels or its effects.

\section{Limitations}

This study has certain limitations. Our study was planned as a descriptive expression analysis of fourteen novel obesity related genes in the animal model of the ZDF rat. Thereby, the aim was on the one hand to identify tissues, in which those genes are expressed, and on the other hand, to reveal differences in the expression levels between ZDF rats and their littermates the ZL rats at an age of 22 weeks. Therefore, this study does not provide information on alterations in different fasting states of the animals, but this might be of interest for future work considering the fact that a high fat diet is known to induce alterations in metabolism and gene expression of adipose tissues [40]. Furthermore as a sole descriptive study in a certain animal model, our results are not simply assignable to other animal models of obesity. This is particularly because ZDF rats are not only obese, but also develop Typ 2 diabetes mellitus. That is why, we cannot exclude that the observed alterations in the gene expression could at least in part be related to the diabetic state of the animals. Additionally, it has to be mentioned, that ZDF rats are homozygous for a defect in the leptin receptor and it is not clear, whether this alone causes alterations in the expression of the investigated obesity genes. The possible impact of insulin resistance and leptin receptor defect could be further investigated by studying Spontaneously Diabetic Torii (SDT) rats as a model of non-obese type 2 diabetes mellitus and a congenic strain of SDT rat with the same leptin receptor defect as the ZDF rats, which are obese and develop type 2 diabetes mellitus [41].

\section{Conclusion}

Thirteen of our fourteen investigated obesity-related genes showed the highest expression in the hypothalamus suggesting a role of these genes predominantly in the central regulation of energy homeostasis. On the other hand the ZDF phenotype accompanies only with expression changes in the adipose tissues, which implies that peripheral mechanisms are important for the development of obesity in ZDF rats. However, further studies 
to reveal the exact function of most of these genes are necessary.

\section{Competing interests}

The authors declare that they have no competing interests.

\section{Acknowledgments}

The authors wish to thank Gabriela Pietrzyk for excellent technical assistance.

\section{Author details}

${ }^{1}$ Klinik und Poliklinik für Innere Medizin II, University of Regensburg, Regensburg, Germany. ${ }^{2}$ Institut für Epidemiologie und Präventivmedizin, University of Regensburg, Regensburg, Germany. ${ }^{3}$ Klinik und Poliklinik für Innere Medizin I, University of Regensburg, Regensburg, Germany. ${ }^{4}$ Klinik und Poliklinik für Innere Medizin II, Franz-Josef-Strauss-Allee 11, University of Regensburg, 93042 Regensburg, Germany.

\section{Authors' contributions}

$\mathrm{PS}, \mathrm{IH}, \mathrm{AL}$ designed research; $\mathrm{PS}, \mathrm{IH}, \mathrm{CB}, \mathrm{AS}, \mathrm{MR}, \mathrm{CB}, \mathrm{DE}, \mathrm{GR}$ and $\mathrm{AL}$ performed research; $C B, A S, G R$ and $D E$ revised the manuscript critically for intellectual content; PS, IH, CB, AS and AL analyzed data; PS and AL wrote the paper. All authors have read and approved submission of the final manuscript.

Received: 28 January 2012 Accepted: 3 May 2012

Published: 3 May 2012

\section{References}

1. Maes $H H$, Neale MC, Eaves $L$ : Genetic and environmental factors in relative body weight and human adiposity. Behav Genet 1997, 27:325-351.

2. Stunkard AJ, Foch TT, Hrubec Z: A twin study of human obesity. JAMA 1986, 256:51-54

3. McCarthy MI: Genomics, type 2 diabetes, and obesity. N Engl J Med 2010, 363:2339-2350.

4. Elks CE, Loos RJ, Sharp SJ, Langenberg C, Ring SM, Timpson NJ, NesS AR, Davey Smith G, Dunger DB, Wareham NJ, Ong KK: Genetic markers of adult obesity risk are associated with greater early infancy weight gain and growth. PLoS Med 2010, 7:e1000284.

5. Heid IM, Jackson AU, Randall JC, Winkler TW, Qi L, Steinthorsdottir V, Thorleifsson G, Zillikens MC, Speliotes EK, Magi R, et al: Meta-analysis identifies 13 new loci associated with waist-hip ratio and reveals sexual dimorphism in the genetic basis of fat distribution. Nat Genet 2010, 42:949-960.

6. Lindgren CM, Heid IM, Randall JC, Lamina C, Steinthorsdottir V, Qi L, Speliotes EK, Thorleifsson G, Willer CJ, Herrera BM, et al: Genome-wide association scan meta-analysis identifies three Loci influencing adiposity and fat distribution. PLoS Genet 2009, 5:e1000508.

7. Ng MC, Tam CH, So WY, Ho JS, Chan AW, Lee HM, Wang Y, Lam VK, Chan JC, Ma RC: Implication of genetic variants near NEGR1, SEC16B, TMEM18, ETV5/DGKG, GNPDA2, LIN7C/BDNF, MTCH2, BCDIN3D/FAIM2, SH2B1, FTO, MC4R, and KCTD15 with obesity and type 2 diabetes in 7705 Chinese. J Clin Endocrinol Metab 2010, 95:2418-2425.

8. Renstrom F, Payne F, Nordstrom A, Brito EC, Rolandsson O, Hallmans G, Barroso I, Nordstrom P, Franks PW: Replication and extension of genome-wide association study results for obesity in 4923 adults from northern Sweden. Hum Mol Genet 2009, 18:1489-1496.

9. Speliotes EK, Willer CJ, Berndt SI, Monda KL, Thorleifsson G, Jackson AU, Allen $\mathrm{HL}$, Lindgren CM, Luan J, Magi R, et al: Association analyses of 249,796 individuals reveal 18 new loci associated with body mass index. Nat Genet 2010, 42:937-948.

10. Thorleifsson G, Walters GB, Gudbjartsson DF, Steinthorsdottir V, Sulem P, Helgadottir A, Styrkarsdottir U, Gretarsdottir S, Thorlacius S, Jonsdottir l, et al: Genome-wide association yields new sequence variants at seven loci that associate with measures of obesity. Nat Genet 2009, 41:18-24.

11. Willer CJ, Speliotes EK, Loos RJ, Li S, Lindgren CM, Heid IM, Berndt SI, Elliott AL, Jackson AU, Lamina C, et al: Six new loci associated with body mass index highlight a neuronal influence on body weight regulation. Nat Genet 2009, 41:25-34.

12. Shin AC, Zheng H, Berthoud HR: An expanded view of energy homeostasis: neural integration of metabolic, cognitive, and emotional drives to eat. Physiol Behav 2009, 97:572-580.
13. Trayhurn P, Bing C, Wood IS: Adipose tissue and adipokines-energy regulation from the human perspective. J Nutr 2006, 136:1935S-1939S

14. Hajer GR, van Haeften TW, Visseren FL: Adipose tissue dysfunction in obesity, diabetes, and vascular diseases. Eur Heart J 2008, 29:2959-2971.

15. Coll AP, Farooqi IS, O'Rahilly S: The hormonal control of food intake. Cell 2007, 129:251-262

16. Frayling TM, Timpson NJ, Weedon MN, Zeggini E, Freathy RM, Lindgren CM, Perry JR, Elliott KS, Lango H, Rayner NW, et al: A common variant in the FTO gene is associated with body mass index and predisposes to childhood and adult obesity. Science 2007, 316:889-894.

17. Tung YC, Yeo GS: From GWAS to biology: lessons from FTO. Ann N Y Acad Sci 2011, 1220:162-171.

18. Tung YC, Ayuso E, Shan X, Bosch F, O'Rahilly S, Coll AP, Yeo GS: Hypothalamic-specific manipulation of Fto, the ortholog of the human obesity gene FTO, affects food intake in rats. PLoS One 2010, 5:e8771.

19. Cone RD: The Central Melanocortin System and Energy Homeostasis. Trends Endocrinol Metab 1999, 10:211-216.

20. Ellacott KL, Cone RD: The central melanocortin system and the integration of short- and long-term regulators of energy homeostasis. Recent Prog Horm Res 2004, 59:395-408.

21. Huszar D, Lynch CA, Fairchild-Huntress V, Dunmore JH, Fang Q, Berkemeier LR, Gu W, Kesterson RA, Boston BA, Cone RD, et al: Targeted disruption of the melanocortin-4 receptor results in obesity in mice. Cell 1997, 88:131-141.

22. Fan W, Boston BA, Kesterson RA, Hruby VJ, Cone RD: Role of melanocortinergic neurons in feeding and the agouti obesity syndrome. Nature 1997, 385:165-168.

23. Ellacott KL, Cone RD: The role of the central melanocortin system in the regulation of food intake and energy homeostasis: lessons from mouse models. Philos Trans R Soc Lond B Biol Sci 2006, 361:1265-1274.

24. Nagahara AH, Tuszynski MH: Potential therapeutic uses of BDNF in neurological and psychiatric disorders. Nat Rev Drug Discov 2011, 10:209-219.

25. Xu B, Goulding EH, Zang K, Cepoi D, Cone RD, Jones KR, Tecott LH, Reichardt LF: Brain-derived neurotrophic factor regulates energy balance downstream of melanocortin-4 receptor. Nat Neurosci 2003, 6:736-742.

26. Almen MS, Jacobsson JA, Shaik JH, Olszewski PK, Cedernaes J, Alsio J, Sreedharan S, Levine AS, Fredriksson R, Marcus C, Schioth HB: The obesity gene, TMEM18, is of ancient origin, found in majority of neuronal cells in all major brain regions and associated with obesity in severely obese children. BMC Med Genet 2010, 11:58

27. Dutta S, Dawid IB: Kctd15 inhibits neural crest formation by attenuating Wnt/beta-catenin signaling output. Development 2010, 137:3013-3018.

28. Marg A, Sirim P, Spaltmann F, Plagge A, Kauselmann G, Buck F, Rathjen FG, Brummendorf $T$ : Neurotractin, a novel neurite outgrowth-promoting Ig-like protein that interacts with CEPU-1 and LAMP. J Cell Biol 1999, 145:865-876.

29. Schafer M, Brauer AU, Savaskan NE, Rathjen FG, Brummendorf T: Neurotractin/kilon promotes neurite outgrowth and is expressed on reactive astrocytes after entorhinal cortex lesion. Mol Cell Neurosci 2005, 29:580-590.

30. Occhi G, Rampazzo A, Beffagna G, Antonio Danieli G: Identification and characterization of heart-specific splicing of human neurexin 3 mRNA (NRXN3). Biochem Biophys Res Commun 2002, 298:151-155.

31. Walley AJ, Jacobson P, Falchi M, Bottolo L, Andersson JC, Petretto E, Bonnefond A, Vaillant E, Lecoeur C, Vatin V, et al: Differential coexpression analysis of obesity-associated networks in human subcutaneous adipose tissue. Int J Obes (Lond) 2012, 36:137-147.

32. Gelb BD: Char Syndrome. GeneReviews [Internet] Seattle (WA): University of Washington, Seattle; 1993-2003 Aug 15 [updated 2008 Mar 19] 1993.

33. Zhao F, Bosserhoff AK, Buettner R, Moser M: A heart-hand syndrome gene: tfap $2 \mathrm{~b}$ plays a critical role in the development and remodeling of mouse ductus arteriosus and limb patterning. PLOS ONE 2011, 6:e22908.

34. Ugi S, Nishio Y, Yamamoto H, Ikeda K, Kobayashi M, Tsukada S, Kondo M, Morino K, Obata T, Yoshizaki T, et al: Relation of the expression of transcriptional factor TFAP2B to that of adipokines in subcutaneous and omental adipose tissues. Obesity (Silver Spring) 2010, 18:1277-1282.

35. Yeung E, Qi L, Hu FB, Zhang C: Novel abdominal adiposity genes and the risk of type 2 diabetes: findings from two prospective cohorts. Int I Mol Epidemiol Genet 2011, 2:138-144.

36. Morrow CM, Hostetler CE, Griswold MD, Hofmann MC, Murphy KM, Cooke PS, Hess RA: ETV5 is required for continuous spermatogenesis in adult mice and may mediate blood testes barrier function and testicular immune privilege. Ann N Y Acad Sci 2007, 1120:144-151. 
37. Leibowitz-Amit R, Tsarfaty G, Abargil Y, Yerushalmi GM, Horev J, Tsarfaty I: Mimp, a mitochondrial carrier homologue, inhibits Met-HGF/SF-induced scattering and tumorigenicity by altering Met-HGF/SF signaling pathways. Cancer Res 2006, 66:8687-8697.

38. Zaltsman Y, Shachnai L, Yivgi-Ohana N, Schwarz M, Maryanovich M, Houtkooper RH, Vaz FM, De Leonardis F, Fiermonte G, Palmieri F, et al: MTCH2/MIMP is a major facilitator of tBID recruitment to mitochondria. Nat Cell Biol 2010, 12:553-562.

39. Kulyte A, Ryden M, Mejhert N, Dungner E, Sjolin E, Arner P, Dahlman I: MTCH2 in Human White Adipose Tissue and Obesity. J Clin Endocrinol Metab 2011, 96:1661-1665.

40. Razny U, Kiec-Wilk B, Wator L, Polus A, Dyduch G, Solnica B, Malecki M, Tomaszewska R, Cooke JP, Dembinska-Kiec A: Increased nitric oxide availability attenuates high fat diet metabolic alterations and gene expression associated with insulin resistance. Cardiovasc Diabetol 2011, 10:68.

41. Masuyama T, Katsuda Y, Shinohara M: A novel model of obesity-related diabetes: introgression of the Lepr $(\mathrm{fa})$ allele of the Zucker fatty rat into nonobese Spontaneously Diabetic Torii (SDT) rats. Exp Anim 2005, 54:13-20.

doi:10.1186/1475-2840-11-48

Cite this article as: Schmid et al: Expression of fourteen novel obesityrelated genes in zucker diabetic fatty rats. Cardiovascular Diabetology 2012 11:48

\section{Submit your next manuscript to BioMed Central and take full advantage of:}

- Convenient online submission

- Thorough peer review

- No space constraints or color figure charges

- Immediate publication on acceptance

- Inclusion in PubMed, CAS, Scopus and Google Scholar

- Research which is freely available for redistribution 